\title{
On polar ovals in abelian projective planes
}

\author{
Kei Yuen Chan Hiu Fai Law Philip P.W. Wong
}

\begin{abstract}
A condition is introduced on the abelian difference set $\mathcal{D}$ of an abelian projective plane of odd order so that the oval $2 \mathcal{D}$ is the set of absolute points of a polarity, with the consequence that any such abelian projective plane is Desarguesian.
\end{abstract}

Keywords: ovals, polarity, cyclic difference sets, projective planes

MSC 2000: 05B10, 05B25, 51E15, 51E21

\section{Introduction}

A projective plane is Desarguesian if any two centrally perspective triangles are axially perspective. It is well-known that a Desarguesian plane can be coordinatized by a division ring. Since any finite division ring is a Galois field, the order of a finite Desarguesian projective plane is a prime power. Although nonDesarguesian planes exist, all known cases have order a prime power. This leads to the following famous long-standing conjecture:

Conjecture 1.1. Any finite projective plane is of prime power order.

In terms of number theoretic properties, the most general result about the conjecture is the Bruck-Ryser-Chowla Theorem [7, 8] which states that if a projective plane of order $n$ exists and $n \equiv 1,2(\bmod 4)$, then $n$ is a sum of two squares. The first case not ruled out by the result is order 10 . The nonexistence of a projective plane of order 10 has been shown by Lam, Thiel and Swiercz [18] by exhaustive computer search. The next general case, which is 12, remains open.

A cyclic projective plane (CPP) is a finite projective plane which admits a cyclic Singer group of collineations, i.e. a collineation group which is sharply transitive on points. Singer [24] has proven that any finite Desarguesian plane is a cyclic projective plane. The converse is another long-standing conjecture: 


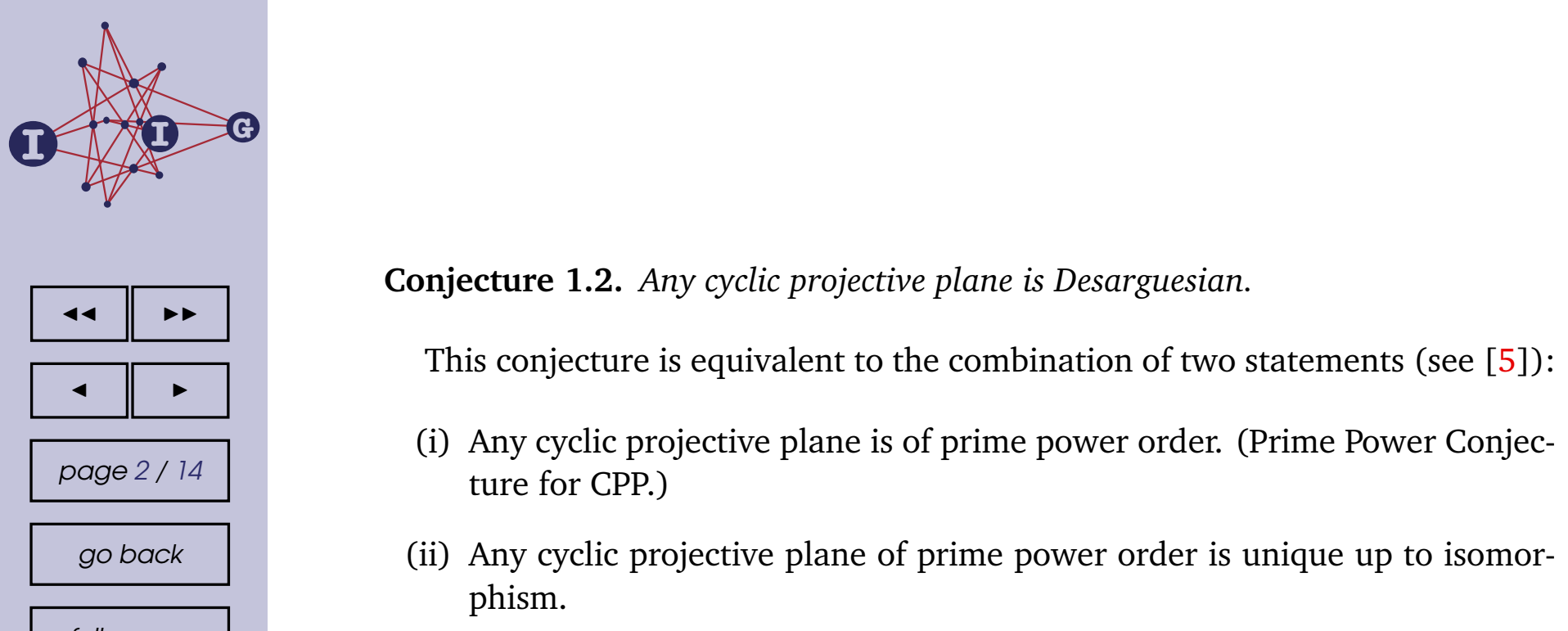

full screen

close quit

ACADEMIA PRESS

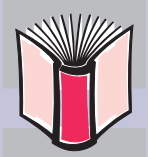

$\widehat{\underline{\text { IIIIII }}}$ UNIVERSITEIT GENT

Statement (i) has been verified up to order $2 \times 10^{9}$ in [2, 9]. Statement (ii) has been first verified for the order $m$ and $m^{2}$ by Bruck [5], where $m=$ $2,3,5,7,8,9$. Recently, Law and Wong [19] have extended Bruck's result to $m=11,16$. Furthermore, Huang and Schmidt [14] have shown that for $n<41$ and for $n \in\{121,125,128,169,256,1024\}$, every CPP of order $n$ is Desarguesian.

More generally, a finite projective plane which admits an abelian Singer group of collineations is called an abelian projective plane (APP). In this paper, we isolate a mild necessary and sufficient condition, configurational in nature, for an APP of odd order to be Desarguesian. Essential to our approach is the result of Ott [21] together with its generalization to the abelian case by Ho [13], and the classic characterization of the multiplier group by Bruck [4], which combine to give the conclusion that an APP $\pi$ with abelian Singer group $G$ is either Desarguesian or $G$ is normal in the full automorphism group $\operatorname{Aut}(\pi)$. We shall construct a polarity on an odd order APP satisfying our condition so that its composition with the Hall polarity associated to an APP defines a collineation which is not in the normalizer of $G$ in $\operatorname{Aut}(\pi)$.

We shall be using the techniques of abelian difference sets. As is wellknown, two cyclic projective planes are isomorphic if and only if the associated cyclic difference sets are equivalent. Furthermore, this result extends to abelian groups by Jungnickel [16]. Accordingly, our configurational condition is given in terms of the difference set defining the APP under investigation. Our proofs depend essentially on the classification of points and lines of a plane with respect to ovals and the use of multipliers. For consistency in notation and ease of reference, we have included the discussion of some basic results in the following sections.

\section{Arcs and ovals in an abelian projective plane}

In this section, we study arcs and ovals and use them to classify the points and lines in an abelian projective plane. 
We begin by working in a finite projective plane and then later specialize to an abelian one. Let $\pi$ be a finite projective plane. An arc of lines (respectively points) is a set of lines (respectively points) which are in general position. An oval is an arc whose cardinality is the largest among all arcs. Note that this definition of an oval is different from the usual one which is given as $q+1$ lines in general position in a plane of order $q$ (see [15], cfr. also [11].)

Definition 2.1. Given an arc of lines $\mathcal{A}$ of $\pi$, a point is called, respectively, a zero point, single point, double point if it is incident on, respectively, 0,1,2 lines in $\mathcal{A}$. The sets of zero points, single points and double points are denoted respectively by $Z, S, D$.

Definition 2.2. Given an arc of lines $\mathcal{A}$ of $\pi$, a line is called, respectively, a zero line, single line, double line if it is incident on, respectively, $0,1,2$ single points of $\mathcal{A}$. The sets of zero lines, single lines and double lines are denoted respectively by $\mathcal{Z}, \mathscr{S}, \mathcal{D}$.

We have the usual dual version of the above definitions for a given arc of points.

We first discuss the size and content of an oval of lines. However, all results hold if we consider points instead.

Since any line $l$ has $q+1$ points and distinct lines of an arc containing $l$ must intersect $l$ at distinct points, it follows that the cardinality of an oval cannot exceed $q+2$. On the other hand, if there are $q+2$ lines in the oval, then each line in it contains only double points. It follows that a line not in the oval must intersect the lines of the oval in $(q+2) / 2$ double points, and so $q$ is even. In fact, it is a remarkable result of Qvist [23] that any arc of $q+1$ lines in a projective plane of even order $q$ can be extended to an oval of $q+2$ lines.

Now suppose $\mathcal{O}$ is an oval of $q+1$ lines in a plane $\pi$ of odd order. It is easy to see that each line in $\mathcal{O}$ has 1 single point and $q$ double points, and each line outside $\mathcal{O}$ has $s$ single points and $d$ double points where $s+2 d=q+1$. In particular, $s \neq 1$ and the set of single lines $\mathscr{S}=\mathcal{O}$. Thus, we may write $\mathcal{O}=\left\{l_{0}, l_{1}, \ldots, l_{q}\right\}$, and the set of single points $S=\left\{P_{0}, P_{1}, \ldots, P_{q}\right\}$, where $P_{i}$ is on $l_{i}, i=0,1, \ldots, q$, and $l_{i}$ is the only single line with respect to $\mathcal{O}$ on $P_{i}$. Counting the lines on $P_{i}$ we conclude that $P_{i} . P_{j}$, where $j=0,1, \ldots, \hat{i}, \ldots, q$, are $q$ distinct lines, so that $S$ is an arc of points. It follows that any line in $\pi$ contains at most two single points.

We summarize the above discussion on planes of odd order in the following structure theorem (cfr. $[11,12]$.)

Theorem 2.3. Let $\mathcal{O}$ be an oval of $q+1$ lines in a projective plane $\pi$ of odd order $q$. The points and lines of $\pi$ are classified with respect to $\mathcal{O}$ as follows: 

As for the double points and double lines, they must also correspond in a one-to-one manner under $\beta$. Indeed, the double point $d_{i}+d_{j}$ is the intersection of the single lines $\mathcal{D}_{q}+d_{i}$ and $\mathcal{D}_{q}+d_{j}$, and since $\beta$ is a correlation, $\left(d_{i}+d_{j}\right)^{\beta}=$ $\left(\mathcal{D}_{q}+d_{i}\right)^{\beta} \cdot\left(\mathcal{D}_{q}+d_{j}\right)^{\beta}=2 d_{i} .2 d_{j}$, which is a double line. Thus, under the polarity $\beta$, the double points and double lines correspond as follows:

$$
\beta: d_{i}+d_{j} \longleftrightarrow 2 d_{i} \cdot 2 d_{j}
$$

for $i, j=0,1, \ldots, q, i \neq j$. Note that the correspondence is one-to-one since $2 \mathcal{D}_{q}$ is an oval.

Now let $Z$ be a zero point. We study the line $Z^{\beta}$. Consider the pencil of lines on $Z$. Since $\beta$ is a correlation, the $v=(q+1) / 2$ double lines $2 d_{i^{\prime}} \cdot 2 d_{i^{\prime \prime}}, i=$ $1,2, \ldots, v$, on $Z$ must correspond under $\beta$ to the double points $d_{i^{\prime}}+d_{i^{\prime \prime}}, i=$ $1,2, \ldots, v$, on $Z^{\beta}$. Since all the single points have been accounted for, these double points are all the double points on $Z^{\beta}$, and so $Z^{\beta}$ must be a zero line. If $Z^{\prime}$ is another zero point, then $Z^{\prime \beta}$ cannot be the same as $Z^{\beta}$ as all double points on $Z^{\beta}$ have been occupied by the images under $\beta$ of the double lines on $Z$. Therefore the zero points and zero lines are also in one-to-one correspondence under $\beta$ given by

$$
\beta: Z \longleftrightarrow\left(2 d_{1^{\prime}} \cdot 2 d_{1^{\prime \prime}}\right)^{\beta} \ldots \ldots .\left(2 d_{v^{\prime}} \cdot 2 d_{v^{\prime \prime}}\right)^{\beta}
$$

where $2 d_{i^{\prime}} .2 d_{i^{\prime \prime}}, i=1,2, \ldots, v$, are the double lines on $Z$.

We return to the oval $2 \mathcal{D}_{q}$. We have shown that if there is a polarity $\beta$ whose absolute points are $2 \mathcal{D}_{q}$, then $\beta$ must be defined by (2), (3), and (4). In particular, this imposes the following condition on the configuration of double points and double lines, given in terms of the difference set $\mathcal{D}_{q}$ :

Condition D. If the double lines $2 d_{i} .2 d_{j}, 2 d_{k} .2 d_{l}$, and $2 d_{m} .2 d_{n}$ are concurrent, then the double points $d_{i}+d_{j}, d_{k}+d_{l}$, and $d_{m}+d_{n}$ are collinear.

Condition $\mathbf{D}$ is therefore a necessary condition for the oval $2 \mathcal{D}_{q}$ to be polar. We shall assume that the odd order abelian projective plane $\pi\left(\mathcal{D}_{q}\right)$ satisfies Condition $\mathbf{D}$, define $\beta$ as above, and proceed to study whether $\beta$ thus defined is a polarity. Since by definition $\beta$ is order 2 , we need only check whether $\beta$ is a correlation. It turns out that a further necessary condition is required. Finally, we must also check that the set of absolute points of $\beta$ is indeed $2 \mathcal{D}_{q}$.

Let $P$ be a point and consider its pencil of lines. With respect to $\beta$, we say that a line $l$ in the pencil is well-behaved if $l^{\beta} \in P^{\beta}$. Dually, let $l$ be a line and consider its points. We say that a point $P$ on the line is well-behaved if $P^{\beta} \ni l^{\beta}$. If lines on points and points on lines are always well-behaved then $\beta$ is (by definition) a correlation. 
We shall also say that a pair of point $P$ and line $l$ is well-behaved with respect to $\beta$ if $P \in l$ and $P^{\beta} \ni l^{\beta}$.

Lemma 3.3. The following lines on points and points on lines are well-behaved with respect to $\beta$ :

(i) lines on a single point, and points on a single line,

go back

full screen

close

quit

(ii) single and zero lines on a double point, and single and zero points on a double line,

(iii) double lines on a zero point, and double points on a zero line.

Proof. Immediate consequences of the definition. To illustrate, consider the case of a zero line $z_{i}$ on a double point $d_{a}+d_{b}$. We show $z_{i}{ }^{\beta} \in\left(d_{a}+d_{b}\right)^{\beta}=2 d_{a} .2 d_{b}$. Now $z_{i}{ }^{\beta}$ is by definition the zero point where the images under $\beta$ of the double lines on it define by their collinearity the line $z_{i}$. Since the double point $d_{a}+d_{b}$ is on $z_{i}$, the double line $2 d_{a} .2 d_{b}$ must be on $z_{i}{ }^{\beta}$, as we wished.

Lemma 3.4. The double lines on a double point are well-behaved with respect to $\beta$.

Proof. Let $u=(q-1) / 2$. Let $2 d_{i^{\prime}} \cdot 2 d_{i^{\prime \prime}}, i=1,2, \ldots, u$, be the double lines on the double point $d_{a}+d_{b}$. We show $\left(2 d_{i^{\prime}} .2 d_{i^{\prime \prime}}\right)^{\beta} \in\left(d_{a}+d_{b}\right)^{\beta}$ for each $i$, i.e. $d_{i^{\prime}}+d_{i^{\prime \prime}} \in 2 d_{a} .2 d_{b}$. By Condition $\mathbf{D}, d_{i^{\prime}}+d_{i^{\prime \prime}}, i=1,2, \ldots, u$, are collinear on a line $l$. If $l$ is a double line, then it must be $2 d_{a} .2 d_{b}$, since all the remaining $2 u=q-1$ single points have been accounted for by the $u$ double points $d_{i^{\prime}}+d_{i^{\prime \prime}}$ on $l$, and we are done. It remains to show that $l$ can neither be a single nor a zero line. If $l$ were a single line, then the single point on it must be either $2 d_{a}$ or $2 d_{b}$ by counting as before, so that $l$ is either $\mathcal{D}_{q}+d_{a}$ or $\mathcal{D}_{q}+d_{b}$. By comparing the double points on $l$ with those on these two single lines it is clear that $l$ can be neither of these two. If $l$ were a zero line, then $l$ corresponds under $\beta$ to the zero point whose double lines must include those corresponding to $d_{i^{\prime}}+d_{i^{\prime \prime}}, i=1,2, \ldots, u$, i.e. $2 d_{i^{\prime}} .2 d_{i^{\prime \prime}}, i=1,2, \ldots, u$. But these lines are concurrent on a double point, namely, $d_{a}+d_{b}$. So $l$ cannot be a zero line either.

Lemma 3.5 (Converse of Condition D). If the double points $d_{i}+d_{j}, d_{k}+$ $d_{l}$, and $d_{m}+d_{n}$ are collinear, then the double lines $2 d_{i} .2 d_{j}, 2 d_{k} .2 d_{l}$, and $2 d_{m} .2 d_{n}$ are concurrent.

Proof. Let $l$ be the line of collinearity of the double points $d_{i}+d_{j}, d_{k}+d_{l}$ and $d_{m}+d_{n}$. Suppose $l$ is a double line, say, $2 d_{a} \cdot 2 d_{b}$. Consider the double point $\left(2 d_{a} \cdot 2 d_{b}\right)^{\beta}=d_{a}+d_{b}$. Let $2 d_{p^{\prime}} \cdot 2 d_{p^{\prime \prime}}, p=1,2, \ldots, u$, be the double lines on 



\section{Multipliers, conics, and Desarguesian planes}

In this section we show that an abelian projective plane satisfying the hypotheses of Theorem 3.11 is Desarguesian. We shall deduce this from the result of Ott [21] with its generalization by Ho [13], and the classic characterization of the multiplier group by Bruck [4], using the polarity $\beta$ provided by Theorem 3.11. Furthermore, we shall use the fact that in a Desarguesian plane of odd order $q$, a classical unital can never contain a conic. It follows that the set of absolute points of the polarity $\beta$, which contains the oval $2 \mathcal{D}_{q}$ (hence a conic by Segre's Theorem, [25]), is equal to $2 \mathcal{D}_{q}$.

Let $\pi\left(\mathcal{D}_{q}\right)$ be an abelian projective plane of odd order $q$, with abelian Singer group $G$ and abelian difference set $\mathcal{D}_{q}=\left\{0, d_{1}, d_{2}, \ldots, d_{q}\right\}$ of $G$. Suppose the conditions of Theorem 3.11 are satisfied, so that we have a polarity $\beta$ satisfying (2), (3), and (4). Let $\alpha$ be the Hall polarity given by (1), and consider the collineation $\alpha \beta$.

Lemma 4.1. The collineation $\alpha \beta$ is not in the normalizer $N(G)$ of $G$ in the full automorphism group $\operatorname{Aut}\left(\pi\left(\mathcal{D}_{q}\right)\right)$.

Proof. Suppose $\alpha \beta \in N(G)$. Let $d_{i} \in \mathcal{D}_{q} \backslash\{0\}$. Then $\alpha \beta d_{i}(\alpha \beta)^{-1}=h_{i}$ for some $h_{i} \in G$. Since $\alpha$ and $\beta$ are polarities, we have $\alpha \beta d_{i}{ }^{2} \beta \alpha=h_{i}{ }^{2}$. Evaluating at 0 we obtain from the definitions that $-d_{i}=\alpha\left(\mathcal{D}_{q}+d_{i}\right)=\alpha \beta\left(2 d_{i}\right)=2 h_{i}$. So $h_{i}=-d_{i} / 2$. Returning to the original equation and evaluating at 0 we obtain $\alpha \beta d_{i} \beta \alpha(0)=\alpha \beta\left(d_{i}\right)=-d_{i} / 2$. By the definition of $\alpha$ this gives $\beta\left(d_{i}\right)=$ $\mathcal{D}_{q}+d_{i} / 2$. Since $\beta\left(d_{i}\right)=\beta\left(0+d_{i}\right)$ is by definition the line $0.2 d_{i}$, there exist $d_{j}, d_{k} \in \mathcal{D}_{q}$ such that $0=d_{j}+d_{i} / 2$ and $2 d_{i}=d_{k}+d_{i} / 2$. So $-d_{i} / 2,3 d_{i} / 2 \in \mathcal{D}_{q}$. But then $0-\left(-d_{i} / 2\right)=3 d_{i} / 2-d_{i}$, giving two expressions for $d_{i} / 2$ as differences of elements of $\mathcal{D}_{q}$ and contradicting the definition of a difference set.

In view of Theorem 4.3 given below, Lemma 4.1 implies that $\pi\left(\mathcal{D}_{q}\right)$ is Desarguesian. Before stating the result formally, we wish to describe an alternate approach in which more emphasis is put on the role played by the multipliers. We shall need the following results.

Theorem 4.2 ([4]). Let $\pi\left(\mathcal{D}_{q}\right)$ be an abelian projective plane with an abelian Singer group $G$. Denote by $M$ the group of all multipliers of $\mathcal{D}_{q}$. Then $M G=$ $N(G)$, where $N(G)$ is the normalizer of $G$ in the full automorphism group of $\pi\left(D_{q}\right)$. Moreover, $N(G) / G$ is isomorphic to $M$.

Theorem 4.3 ([13], [3, Theorem VI 7.4]). Let $\pi\left(\mathcal{D}_{q}\right)$ be an abelian projective plane with an abelian Singer group $G$. Then either $\pi\left(\mathcal{D}_{q}\right)$ is Desarguesian or $G$ is a normal subgroup of the full automorphism group of $\pi\left(\mathcal{D}_{q}\right)$. 
Note that Theorem 4.3 is a consequence of Theorem 4.2 and the result of Ho [13], which states that an abelian projective plane is Desarguesian if it admits another abelian Singer group. Indeed, since $N(G)=M G$ by Theorem 4.2, if $\pi\left(\mathcal{D}_{q}\right)$ is Desarguesian then it has elations and these are clearly not in $N(G)$ and so $G$ is not normal in $\operatorname{Aut}\left(\pi\left(\mathcal{D}_{q}\right)\right)$. On the other hand, if $\gamma \in \operatorname{Aut}\left(\pi\left(\mathcal{D}_{q}\right)\right) \backslash G$, then $\gamma G \gamma^{-1} \neq G$ and by the result of Ho [13], $\pi\left(\mathcal{D}_{q}\right)$ is Desarguesian.

We saw in Section 2 that $-\mathcal{D}_{q}$ is always an arc, and if $q$ is odd then $2 \mathcal{D}_{q}$ is an oval. Thus -1 is never a multiplier, nor is 2 for $q$ odd. On the other hand, by the Multiplier Theorem [10, 22], 2 is a multiplier for $q$ even. We next study -2 .

Lemma 4.4. -2 is not a multiplier for any abelian difference set $\mathcal{D}_{q}$ of arbitrary order $q$.

Proof. First suppose $q$ is even. Since 2 is a multiplier, $-2 \mathcal{D}_{q}=-\mathcal{D}_{q}+g$ for some $g \in G$, which is an arc, and so -2 is not a multiplier. Next suppose $q$ is odd. Take $d_{i} \in \mathcal{D}_{q}$ such that $d_{i} \neq 0$. If -2 were a multiplier, we may assume that $\mathcal{D}_{q}$ is invariant under -2 ([20]). Then $-2 \mathcal{D}_{q}=\mathcal{D}_{q}$, so that $-2 d_{i}=d_{j}$ and $-2 d_{k}=d_{i}$ for some $d_{j}, d_{k} \in \mathcal{D}_{q}$. Then $d_{i}+d_{j}=2 d_{k}$. Now $d_{j} \neq d_{i}$ since $d_{i} \neq 0$, and so $d_{i}+d_{j}$ is a double point. However, $2 d_{k}$ is a single point, giving a contradiction.

We are now ready to put the significance of the existence of the polarity $\beta$ of Section 3 in the following light.

Lemma 4.5. Let $q$ be odd. An abelian projective plane $\pi\left(\mathcal{D}_{q}\right)$ with an abelian group $G$ is Desarguesian if and only if it admits a polarity satisfying (2).

Proof. If $\pi\left(D_{q}\right)$ is Desarguesian, then since $2 \mathcal{D}_{q}$ is an oval and $q$ is odd, $2 \mathcal{D}_{q}$ is a conic by Segre's Theorem [25]. Hence, $2 \mathcal{D}_{q}$ is the set of absolute points of an orthogonal polarity. Conversely, we assume the contrary and obtain a contradiction. Suppose $\pi\left(\mathcal{D}_{q}\right)$ is not Desarguesian, and let $\beta$ be a polarity satisfying the hypothesis. Consider the collineation $\alpha \beta$, where $\alpha$ is the Hall polarity given by (1). By Theorem 4.3, Aut $\left(\pi\left(\mathcal{D}_{q}\right)\right) \subset N(G)$ and so $\alpha \beta \in N(G)$, where $N(G)$ is the normalizer of $G$ in the automorphism group $\operatorname{Aut}\left(\pi\left(\mathcal{D}_{q}\right)\right)$ of $\pi\left(\mathcal{D}_{q}\right)$. Then Theorem 4.2 implies $\alpha \beta \in M G$ and so for $x \in \pi\left(\mathcal{D}_{q}\right), \alpha \beta(x)=a(x)+g$, for some multiplier $a \in M$ and $g \in G$. Since $\alpha \beta\left(2 d_{i}\right)=\alpha\left(\mathcal{D}_{q}+d_{i}\right)=-d_{i}$ for all $i$, it follows that $a\left(2 d_{i}-2 d_{j}\right)=a\left(2 d_{i}\right)-a\left(2 d_{j}\right)=\alpha \beta\left(2 d_{i}\right)-\alpha \beta\left(2 d_{j}\right)=d_{j}-d_{i}$ for all distinct pairs $i, j$. This covers every element of $G$ and so $a=-\frac{1}{2}$, which is not a multiplier by Lemma 4.4. We have a contradiction and this proves the lemma. 
For our purpose, Lemma 4.5 is an alternative to Lemma 4.1. In other words, with either Lemma, we can add that $\pi\left(\mathcal{D}_{q}\right)$ is Desarguesian to the conclusion in Theorem 3.11.

We have yet to prove that the set of absolute points of $\beta$ is exactly $2 \mathcal{D}_{q}$. However, now that the plane in question is Desarguesian, if the set of absolute points of $\beta$ is larger than $2 \mathcal{D}_{q}$, then $\beta$ is a unitary polarity, its set of absolute points is the set of points of a classical unital $\mathcal{U}$, and the order $q$ of the plane is a square. We now have an oval $2 \mathcal{D}_{q}$, which is a conic, contained in $\mathcal{U}$, in a Desarguesian plane. This is impossible, by the following classical result.

Lemma 4.6. In the Desarguesian plane $P G\left(2, q^{2}\right)$, where $q$ is odd, a conic is not a subset of a classical unital.

Proof. See, for example, [1, Corollary 4.20, p.79].

We have now proven the following result.

Theorem 4.7. Let $\pi\left(\mathcal{D}_{q}\right)$ be an abelian projective plane of odd order $q$ defined by an abelian difference set $\mathcal{D}_{q}=\left\{0, d_{1}, d_{2}, \ldots, d_{q}\right\}$. Let $\beta$ be defined by (2), (3) and (4). Suppose Condition $\mathbf{D}$ holds, and there exists a pair of zero point and zero line well-behaved with respect to $\beta$. Then $2 \mathcal{D}_{q}$ is a polar oval with respect to $\beta$, and $\pi\left(\mathcal{D}_{q}\right)$ is Desarguesian.

Remark 4.8. The sufficient conditions in Theorem 4.7 are all satisfied by a Desarguesian plane and are therefore necessary conditions.

Remark 4.9. Unlike the odd order case, where the single points with respect to an oval of lines themselves form an oval, in the case of a plane of even order $n$, the single points with respect to an arc of $n+1$ lines are collinear. Hence, the study of the analogous problem for the even order case requires the discovery of a different approach. In this connection, in addition to ovals, it is of interest, for planes of square order of both parities, to study the existence and types of unitals embedded in a CPP/APP.

Acknowledgment. The authors would like to thank the referees for their comments and suggestions which led to an improvement of the results.

\section{References}

[1] S. Barwick and G. Ebert, Unitals in projective planes (2008), SpringerVerlag, New York. 
[2] L.D. Baumert and D.M. Gordon, On the existence of cyclic difference sets with small parameters. In: High Primes and Misdemeanours: Lectures in Honour of the 60th Birthday of Hugh Cowie Williams. Fields Inst. Commun. 41 (2004), 61-68.

[3] T. Beth, D. Jungnickel and H. Lenz, Design theory (second edition) (1999), Cambridge University Press, Cambridge.

[4] R.H. Bruck, Difference sets in finite groups, Trans. Amer. Math. Soc. 78 (1955), 464-481.

[5] _ Quadratic extensions of cyclic planes, Combinatorial Analysis, Proceedings of Symposia in Applied Mathematics X (1960), 15-44. American Mathematical Society, Providence, Rhode Island.

[6] Circle geometry in higher dimensions. II. Geom. Ded. 2 (1973), 133-188.

[7] R.H. Bruck and H.J. Ryser, The non-existence of certain finite projective planes, Canadian J. Math. 1 (1949), 88-93.

[8] S. Chowla and H.J. Ryser, Combinatorial problems. Canad. J. Math. 2 (1950), 93-99.

[9] D.M. Gordon, The prime power conjecture is true for $n<2,000,000$. Electron. J. Comb. 1 (1994), R6, 7pp.

[10] M. Jr. Hall, Cyclic projective planes, Duke Math. J. 14 (1947), 1079-1090.

[11] J.W.P. Hirschfeld, Projective geometries over finite fields (second edition) (1998), Clarendon Press, Oxford.

[12] C.Y. Ho, Some remarks on orders of projective planes, planar difference sets and multipliers, Des. Codes Cryptogr. 1 (1991), 69-75.

[13] _ Finite projective planes with transitive abelian collineation groups. J. Algebra 208 (1998), 533-550.

[14] Y. Huang and B. Schmidt, Uniqueness of some cyclic projective planes, Des. Codes Cryptogr. 50 (2009), 253-266.

[15] D.R. Hughes and F.C. Piper, Projective Planes (1973), Springer-Verlag, New York.

[16] D. Jungnickel, The isomorphism problem for abelian projective planes. AAECC 19 (2008), 195-200. 
